

DOI: $10.22144 /$ ctu.jen.2019.012

\title{
Studies on betalains chemistry from the Vietnamese red dragon fruits (Hylocereus polyrhizus) by LC-ESI-MS/MS
}

\author{
Tran Thi Minh Thu ${ }^{1 *}$ and Do Vinh Quang ${ }^{2}$ \\ ${ }^{I}$ Department of Food Technology and Bio-engineering, Can Tho University of Technology, Vietnam \\ ${ }^{2}$ Department of Mechanical Engineering, Can Tho University of Technology, Vietnam \\ ${ }^{*}$ Correspondence:Tran Thi Minh Thu (email: ttmthu@ctuet.edu.vn)
}

\section{Article info.}

Received 30 Aug 2018

Revised 19 Jan 2019

Accepted 29 Mar 2019

\section{Keywords}

Betalains, betacyanins, betaxanthins, Hylocereus polyhizus (red dragon fruits), $L C-E S I-M S / M S$

\begin{abstract}
The principle pigments from Vietnamese red dragon fruits (Hylocereus polyrhizus) were investigated qualitatively the first time by liquid chromatography connected to electrospray ionization tandem mass spectrometry approach (LC-ESI-MS/MS). Both betacyanins and betaxanthins were detected from the crude and purified extracts based on the maximum UV absorbance of $540 \mathrm{~nm}$ and $480 \mathrm{~nm}$, together with the daughter ions at $\mathrm{m} / \mathrm{z}$ 389 and 265, respectively. The main betacyanins were identified as betanin/isobetanin $\left([\mathrm{M}+\mathrm{H}]^{+}\right.$at $\mathrm{m} / \mathrm{z}$ 551), phyllocactin/isophyllocactin (6'O-malonylbetanin, $\left[\mathrm{M}+\mathrm{H}^{+}\right.$at $\mathrm{m} / \mathrm{z}$ 637) and apiosyl-betanin/apiosyl-isobetanin $\left([M+H]^{+}\right.$at $\mathrm{m} / \mathrm{z}$ 683) next to the minor derivative of hylocerenin/isohylocerenin $\left([\mathrm{M}+\mathrm{H}]^{+}\right.$at $\mathrm{m} / \mathrm{z}$ 695), 2'-apiosyl-phyllocactin/2'apiosyl-isophyllocactin $\left([\mathrm{M}+\mathrm{H}]^{+}\right.$at $\mathrm{m} / \mathrm{z}$ 769) and traces of betanidin 5-O$\beta$-sophoroside/isobetanidin 5-O- $\beta$-sophoroside $\left([M+H]^{+}\right.$at $\mathrm{m} / z$ 713). The primary yellow indicaxanthin was also recognized at $\mathrm{m} / \mathrm{z} 309$. These compounds appeared in different ratios in comparison with other Hylocereus polyrhizus cultivar reported before. Their degradation products of decarboxylation and dehydrogenation, namely decarboxy-betanin $\left([\mathrm{M}+\mathrm{H}]^{+}\right.$at $\mathrm{m} / \mathrm{z}$ 507) and neobetanin $\left([\mathrm{M}+\mathrm{H}]^{+}\right.$at $\left.\mathrm{m} / \mathrm{z} 549\right)$ were also found in traces. These artifacts were not found in the fresh fruits and were suspected to be generated through harvesting and processing conditions of high temperature and sunlight.
\end{abstract}

Cited as: Thu, T.T.M. and Quang, D.V., 2019. Studies on betalains chemistry from the Vietnamese red dragon fruits (Hylocereus polyrhizus) by LC-ESI-MS/MS. Can Tho University Journal of Science. 11(1): $87-94$.

\section{INTRODUCTION}

Red dragon fruit or pitahaya is a cactus species belonged to the genus Hylocereus under the Cactaceae family (Caryophyllales order) (Wybraniec and Mizrahi, 2002). This family originated in South America and expanded to tropical/subtropical regions before being commercially cultivated in some countries as Spain,
Mexico, Israel, Taiwan, Malaysia and Vietnam nowadays (He et al., 2012). The plant mainly distributed in Long An, Binh Thuan and Tien Giang provinces and supplied up to $95 \%$ national dragon fruit production (Mac Xuan Hoa et al., 2017). The most dominant Hylocereus polyrhizus cultivated in Vietnam was developed from the Colombian red species and the Vietnamese white species (Hylocereus undatus) through hybridization and 
plant selection. This species has become popular worldwide thanks to their attractive color, fresh taste and rich in nutrition such as vitamin $\mathrm{B}, \mathrm{C}$, minerals, polyphenols and betalains; these micro nutritients in combination make the fruit's distinct sensoria and the healthy low calories commodity (Le et al., 2006). These components contribute to reduction of cholesterol, blood pressure, diabetes and prevent colon cancer; especially the polyphenols and betalains are necessary for neutralizing the free radicals which initiate human cancer (Zainoldin and Baba, 2009). Besides, the significant pigments level (approximately $0.32-0.47 \mathrm{mg} / \mathrm{g}$ flesh depended on cultivars, extraction methods, and measurements) (Phan Thi Thanh Que et al., 2017) makes these fruits a potential cheap-source for colorants extraction.

Betalains are N-heterocyclic colored compounds which are classified into two subclasses of betacyanins and betaxanthins; from which betanin and indicaxanthin were the first betacyanin and betaxanthin being identified (Figure 1) (Stintzing and Carle, 2007). The betanin could be glycosylated at position $\mathrm{C} 5$ or $\mathrm{C} 6$ with typical glycosidic substituents such as glucoses, apiose, and
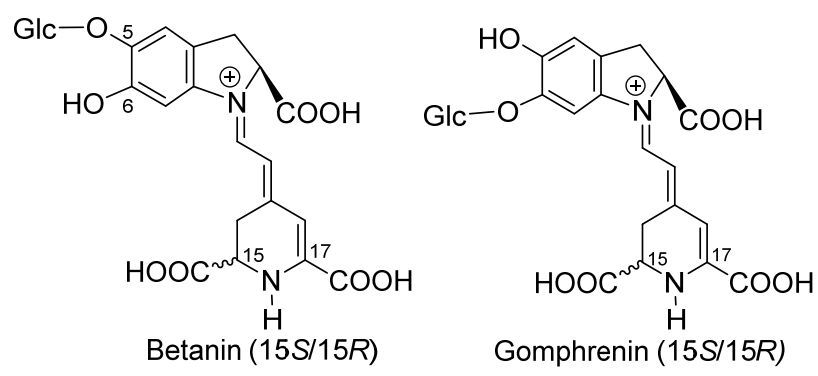

occasionally glucuronic acid for more betacyanin derivatives; these compounds then might be further acylated by the aliphatic or aromatic acid such as malonic, caffeic, p-coumaric, ferulic or sinapic acid (Stintzinget al., 2008) for more structures. Betacyanins display a stereogenic center at $\mathrm{C} 15$, and these stereoisomers are always co-existing (Mabry and Dreiding, 1968). Figure 1 illustrates the chemical configuration of the several typical betalains. Structurally, red dragon fruit pigments consist of both non-acylated and acylated betacyanins, namely betanin, phyllocactin, and hylocerenin in various ratios depending on cultivars. For instance, betanin could account up to $76 \%$, and hylocerenin could contribute $11.7 \%$ of total betacyanin, while the phyllocactin was more dominant than betanin in several varieties (Wybraniec and Mizrahi, 2002). In addition, few minor pigments were also reported from this fruit such as betanidin 5-O- $\beta$-sophoroside, 2 '-apiosylbetanin, sinapoylated apiosyl-betanin, etc. (Fathordoobady et al., 2016). In case of betaxanthin, next to the principle indicaxanthin, other yellow betaxanthins were also detected as $\gamma$-amino butyric acid and so on (Wybraniecet al., 2007).
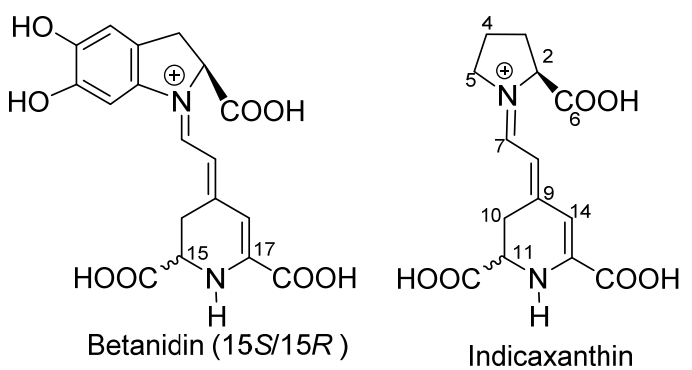

Fig. 1: Betalains typical structures

The stability of dragon fruit pigments depends not only on their structural substitution (Herbach et al., 2006b) but also on the various environmental and processing conditions such as $\mathrm{pH}$, temperature, and the composition of food matrices, which have protective contributions to these colorants (Herbach et al., 2006a). Recently, the dragon fruit is one of the most popular commodities for fresh consumption as well as for processing in Vietnam; however, the high betalain content that could be potential coloring resource has not been discussed intensively, particularly the chemical structures because of the limitation in analysis technology and the high sensitivities of these compounds. This study is aimed to investigate the chemistry characterization of these Vietnamese fruit pigments by applying the highly sensitive detection method of liquid chromatography and electrospray ionization (alternative mode) within a tandem mass spectrometry approach (LC-ESI-MS/MS).

\section{MATERIAL AND METHODS}

\subsection{Material and chemicals}

Red dragon fruits were bought in the local market in Can Tho City; the fresh fruits were then kept at $20^{\circ} \mathrm{C}$ in dark for further investigation.

Acetonitrile (LC-MS and HPLC grade, HoneywellGermany), formic acid (LC-MS grade, SigmaAldrich-Germany), $\mathrm{C} 18$ resin for liquid chromatography (40-63 $\mu \mathrm{m}$, Merck), acetic acid (technical grade, $\geq 99.8 \%$, Sigma-Germany), ethanol (HPLC grade, Sigma-Germany) were used. Nanopure water was purified by the NANOpure Diamond Analytical system $(0.2 \mu \mathrm{m}$ spore, Wilhelm Werner, Leverkusen-Germany). 


\subsection{Sample preparation}

\subsubsection{Pigment extraction}

The frozen fruits were peeled off, and flesh was blended by a kitchen blender before being macerated several times with the Nanopure water $0.7 \%$ acetic acid. The combined extracts were filtered out by cotton and paper filter (MN 165 1/4, $\phi$ 500, Macherey-Nagel-Germany) to remove the residues (flesh, seeds) before being directly lyophilized by freeze drier (Martin Christ Gefriertrocknungsanlagen GmbH-Germany). The dried extracts were kept in the dark at $-20^{\circ} \mathrm{C}$ for further analysis. All extraction steps were done as fast as possible under light protection and at room temperature to prevent betalain degradation.

\subsubsection{Pigments enrichment by reversed phase C18 materials}

C18 solid was washed three times with acetonitrile, then conditioned with aqueous acetic acid $(0.7 \%)$ four times before being incubated with the crude extract within 1 hour. The pigmented resin was rinsed exhaustively with aqueous acetic acid $(0.7 \%)$ to remove un-adsorbed compounds such as sugar and fruit acids. The desorption was done by ethanol:aqueous acetic acid $0.7 \%(\mathrm{v} / \mathrm{v}, 2: 8)$, and the supernatant was freeze-dried (Martin Christ Gefriertrocknungsanlagen $\mathrm{GmbH}$-Germany). The dried extract was kept in the dark at $-20^{\circ} \mathrm{C}$.

\subsubsection{Pigment quantitatively evaluation by $L C$ - ESI-MS/MS}

For the LC: Solvent A: $1 \%$ formic acid $(\mathrm{v} / \mathrm{v})$ in water; solvent $\mathrm{B}$ : $1 \%$ formic acid $(\mathrm{v} / \mathrm{v})$ in acetonitrile. The LC column was Prontosil C18-Aqi column $(250 \mathrm{~mm} \times 2.0 \mathrm{~mm}, 5 \mu, 100 \AA$, Knauer Wissenschaftliche Geräte GmbH, Germany) within an ion-trap-electrospray mass-spectrometer HCTUltra ETD II Bruker Daltonics (Germany) connected with the binary HPLC-pump (model G1312 A, 1100 Series, Agilent TechnologiesGermany). The samples were injected by an autosampler and detector ALS-SL G1329B, FC/ALS therm G1330B, Agilent Technologies (Germany) using linear gradient, namely $99 \% \mathrm{~A}$ as initial conditions ( 0 minute), $80 \%$ A for 20 minutes, $50 \%$ A to 35 minutes, $0 \%$ A to 55 minutes, and $99 \%$ A to 70 minutes.

ESI-MS/MS settings: Alternating ionization mode in the scan range of $m / z 100-1500$ was used with the "ultra"-mode with a mass scanning rate of 26.000 $\mathrm{m} / \mathrm{z}$ per second. Drying gas was nitrogen (11.0 $\mathrm{L} / \mathrm{min}, 330^{\circ} \mathrm{C}$ ) and nebulizer pressure was $60 \mathrm{psi}$. High ionization voltage (HV) capillary was -3700 $\mathrm{V}, \mathrm{HV}$ end plate offset $-500 \mathrm{~V}$, trap drive 72.1 , octopole radiofrequency (RF) amplitude $187.1 \mathrm{Vpp}$, lens $260.0 \mathrm{~V}$, Cap Ex $115.0 \mathrm{~V}$, maximum accumulation time $200 \mathrm{~ms}$. Averages of five spectra, trap drive level $120 \%$, target mass range: $\mathrm{m} / z$ 500, compound stability $80 \%$, Smart ICC target 100000 , ICC charge control "on" and smart parameter setting "active". Three most intensive precursor ions were selected for fragmentation. The MS/MS fragmentation amplitude value was $1 \mathrm{~V}$. The collected data was processed by Data analysis version 3.0 (Bruker Daltonics, Germany) software.

The injected samples were prepared by diluting the dried pigment (powder) with water at concentration of $0.5-1 \mathrm{mg}$ dried extract $/ \mathrm{ml}$ depending on the concentration of the powders, and the injected volume was ranged from $10-15 \mu \mathrm{L}$ depending on the MS/MS ion intensity (trial and error until significant ion intensity was reached).

\section{RESULTS AND DISCUSSION}

\subsection{LC- ESI-MS/MS evaluation of crude extract}

The crude extract was screen by LC-ESI-MS/MS for target pigments and derivatives; LC chromatogram displays in Figure 2. Retention time, UV absorbance, MS spectra or fragmentation patterns and neutral losses $(\Delta \mathrm{m} / \mathrm{z})$ were used for qualitative identification of betalains (Mata et al., 2016), in which the most intensive and well separated peaks detected from the total ion chromatogram (TIC) were selected as precursors for tandem fragmentation. These results allowed the identification of different betalain derivatives based on the recognized parent and daughter ions $\left([\mathrm{M}+\mathrm{H}]^{+}\right.$ or $[\mathrm{M}-\mathrm{H}]^{-}$) in comparison with standards $\mathrm{MS} / \mathrm{MS}$ fragmentation spectra available in literature. TIC data can also be used to quantitatively evaluate the pigment extract (based on their peak areas in comparison with standard compounds); however, the highly sensitivities of these classes of pigment made standards not stable (not available) that the quantitatively evaluation was usually done by spectrophotometry method, which was not discussed detail in this study.

From the TIC of the crude extract (Figure 2A), several pigments were detected by the retention time, UV absorbance together with ESI-MS/MS characteristic signals $[\mathrm{M}+\mathrm{H}]^{+}$at $\mathrm{m} / z 551$ and 389 such as betanin/isobetanin (1/1', $\mathrm{m} / z$ 551), phyllocactin/isophyllocactin (2/2', $m / z$ 637), 2'apiosyl-betanin (3/3', $m / z$ 683) and hylocerenin/isohylocerenin $(\mathrm{m} / \mathrm{z} 695$, in traces). They are known as the typical betacyanin derivatives which were reported from other Hylocereus cultivars (Singer and Von, 1980; 
Mereddy et al., 2017). Base on the ion intensity, betanin epimers were found overwhelmed the rest of the other derivatives, followed by phyllocactin and 2'-apiosyl-betanin, respectively. Hylocerenin/isohylocerenin, on the other hands, appeared in very low intensity indicating for its low concentration in the crude extract (Figure 2B). Likewise, indicaxanthin epimers $\left(4 / 4^{\prime}, m / z\right.$ at 309$)$ were found as the main yellow betaxanthin from this cultivar.

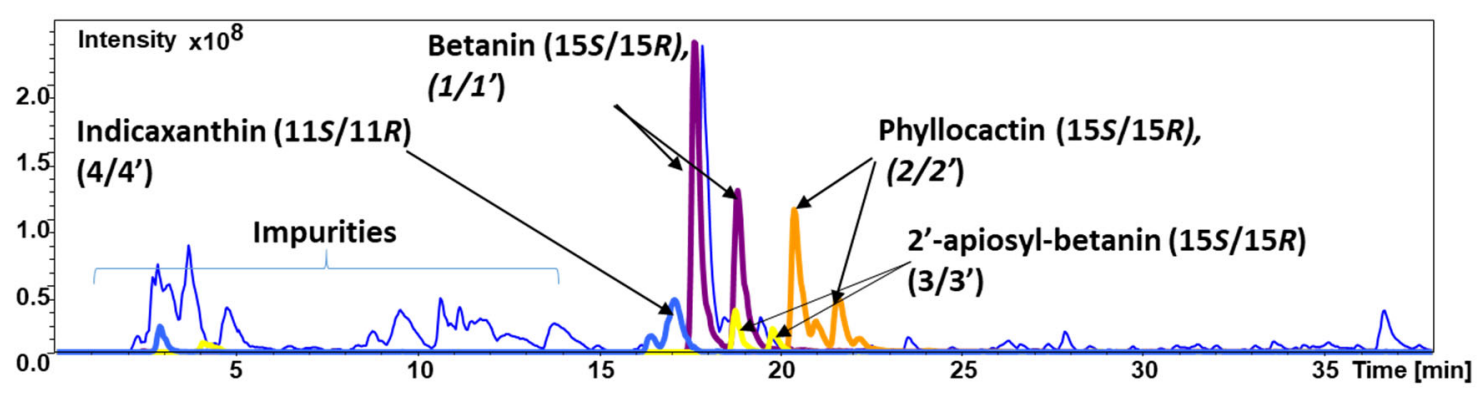

A

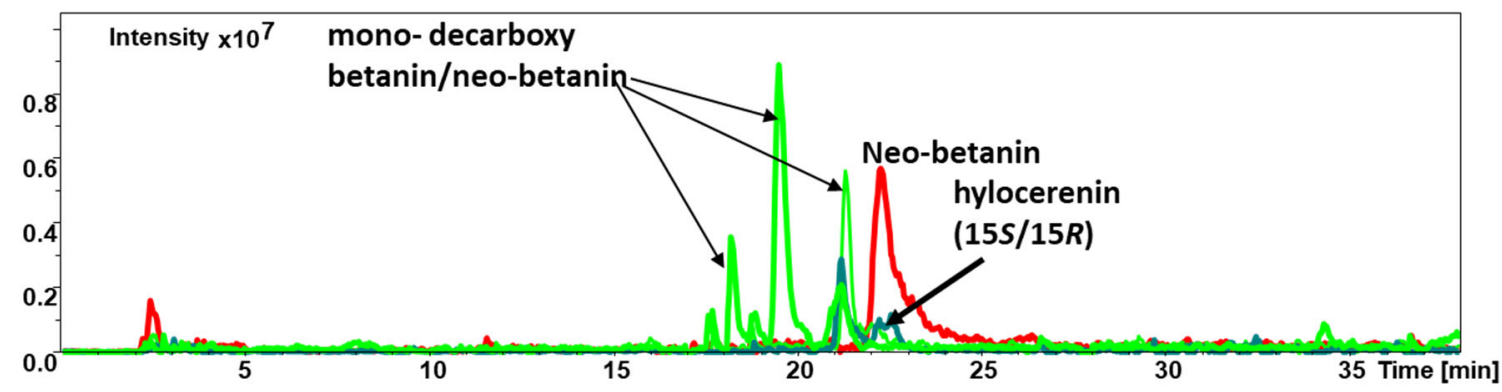

B

Fig. 2: Modified LC chromatogram (or TIC) of Vietnamese red dragon fruitpigment crude extract (A: target pigments; B: degraded pigments)

In addition, the degraded pigments were also recognized from this crude extract in low ion intensities (Figure 2B). These deteriorated compounds namely mono-decarboxy betanin $(\mathrm{m} / \mathrm{z} 507)$, which were found as the most intensive and abundant (degraded) peaks because of their multiple epimers available while neobetanin $(\mathrm{m} / \mathrm{z} 549)$ was rarely detected. These side products were also nominated from previous reports of cacti pigments; they might be generated quickly during extraction or induced by the usage of solvents. Additionally, there were a lot of non-pigmented compounds such as fruity acids, sugars and other metabolites (impurities), which were not in the focus of this study. Although the ESI-MS/MS data showed good ionization capacity, the high concentration of these interfered compounds, particularly the high dose of sugars and pectin substances (data not showed), caused negative effects to the ionization. That could lead to the weak sensitivities of low concentrated pigments detection. Therefore, the $\mathrm{C} 18$ material was used to enrich this crude pigment extract because the resin has high adsorption capacity to betalains. However, this solid material in combination with alcoholic solvents might enhance the degradation of betalains (Von et al., 1974; Wybraniec and Mizrahi, 2005).

\subsection{LC- ESI-MS/MS evaluation of enriched extract}

The LC chromatogram (modified) of the C18 enriched sample is shown in Figure 3. In comparison with the LC chromatogram of crude extract in Figure 1, a majority of interfered compounds were removed successfully by this pre-purification step while low concentrated pigments were intensively accumulated although they were not well detected in the crude extract. Similarly, the high ion intensity and well separated peaks from the chromatogram were selected as precursors for mass spectra evaluation. The peaks assignment and identified pigments/degraded pigments including ESI-MS/MS data from both analyses were summarized in Table 1. 


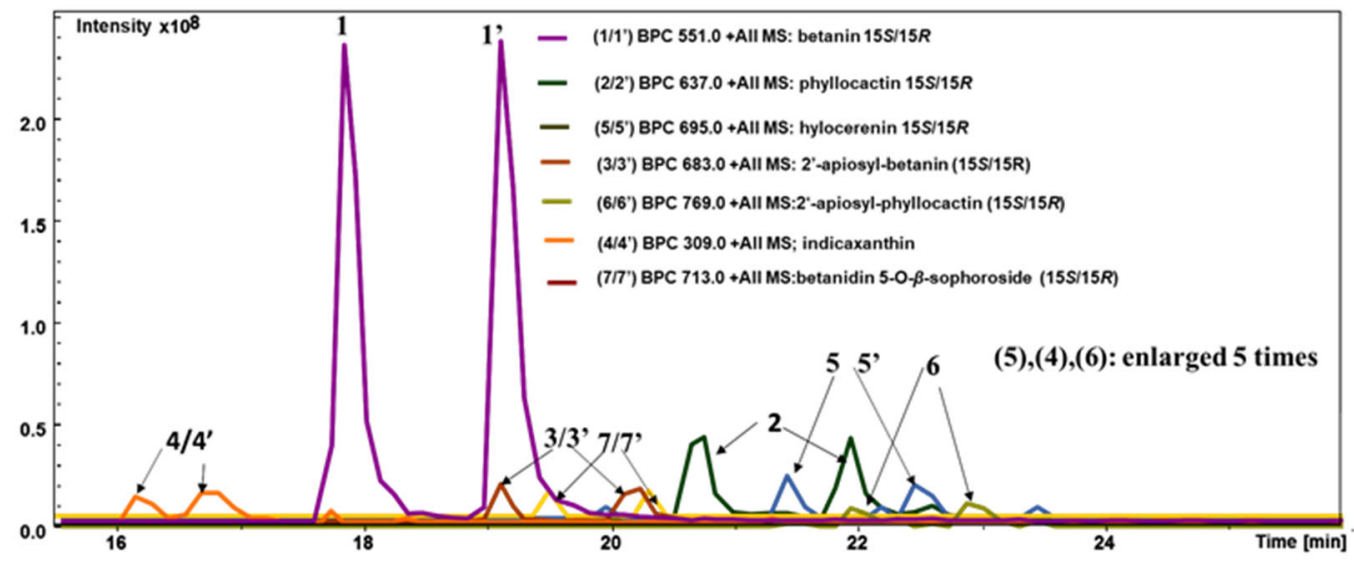

Fig. 3: Modified LC chromatogram of C18 enriched Hylocereus polyhizus pigment extract

Table 1: Characterization of betalains and degraded betalains from Hylocereus polyhizus monitored by LC-ESI-MS/MS (alternative mode)

\begin{tabular}{clrrl}
\hline Number & Name & $\begin{array}{r}\lambda \mathbf{m a x} \\
(\mathbf{n m})\end{array}$ & $\begin{array}{r}\boldsymbol{m} / \boldsymbol{z} \\
{\left[\mathbf{M}+\mathbf{H}^{+}\right.}\end{array}$ & $\mathbf{M S}^{\mathbf{2}} \mathbf{m} / \mathbf{z}$ \\
\hline $1 / 1{ }^{\prime}$ & Betanin $(15 S / 15 R)$ & 538 & 551 & $389(100)$ \\
$2 / 2$ & Phyllocactin $(15 S / 15 R)$ & 536 & 637 & $551(29.26), 389(100)$ \\
$5 / 5$ & Hylocerenin $(15 S / 15 R)$ & 539 & 695 & $551(100), 389(9.46)$ \\
$7 / 7$ & Betanidin 5-O- $\beta$-sophoroside $(15 S / 15 R)$ & 539 & 713 & $551(8.87), 389(81.69)$ \\
3/3' & 2'-apiosyl-betanin $(15 S / 15 R)$ & 538 & 683 & $551(29.26), 389(100)$ \\
$6 / 6$ & 2'-apiosyl-phyllocactin $(15 S / 15 R)$ & 541 & 769 & $683(18.5), 389(100)$ \\
$4 / 4$ & Indicaxanthin & 483 & 309 & $265(100)$ \\
& Mono-decarboxy betanin & 520 & 507 & $345(100)$ \\
& Neobetanin & 520 & 549 & $387(100)$ \\
\hline
\end{tabular}

As can be seen in Figure 3, the purified sample contained clearly six betacyanins derivatives, in which betanin (1) and isobetanin (1') were the principle components showing very high ion intensities. The $15 S$ monomer (betanin) is more polar and eluted lightly earlier than its $15 R$ epimer (isobetanin). Their presences represented by the $[\mathrm{M}+\mathrm{H}]^{+}$ion at $\mathrm{m} / \mathrm{z} 551$ and the cleavage of a glucose unit $(\Delta \mathrm{m} / \mathrm{z}$ 162 ) to the signal at $m / z 389$ (Figure 4A) (Fernández-lópez et al., 2007). Likewise, the signals of phyllocactin/isophyllocactin (2/2', $m / z$ 637) and 2'apiosyl-betanin/2'-apiosyl-isobetanin (3/3', $\mathrm{m} / \mathrm{z}$ $683)$ were recognized through the characteristic ions at $m / z 551,389$. They produced the neutral losses of $\Delta m / z 86$ and 132 indicating malony and apiosyl unit, respectively (Figure 4B and 4C).

Similar to the crude extract, hylocerenin/ isohylocerenin $\left(5 / 5^{\prime}\right)$ - the typical pigments of these fruits - were found very late in the LC chromatogram because of their more lipophilic structures, and appeared in very low ion intensities at $\mathrm{m} / \mathrm{z} 695$ (Figure 5A) (Jerz et al., 2013). Besides, the minor pigment displaying at $m / z 769\left(6 / 6^{\prime}\right)$ was tentatively identified as 2' $2^{6}$-apiosyl-phyllocactin/2'-apiosylisophyllocactin based on these characteristic fragments and the cleavage of both malonyl unit $(\Delta m / z$ $86)$ next to an apiosyl unit $(\Delta m / z$ 132) (Figure 5B). This structure made $6 / 6$ 'the most lipophilic cover the rest of found betacyanins. On the other hands, another minor pigment (7/7', $\mathrm{m} / \mathrm{z} 713$ ) occurred very early in the LC chromatogram represented for the highly polar glucosyl-betanin structure with the additional cleavage of a hexose unit $\Delta \mathrm{m} / z 162$ (Figure $5 \mathrm{C})$. This compound was betanidin 5-O- $\beta$-sophoroside which was detected previously in opuntia fruits (Jerz et al., 2013).

Indicaxanthin/isoindicaxanthin $\left(4 / 4^{\prime}, \mathrm{m} / \mathrm{z}\right.$ at 309) were found as the sole abundant betaxanthin even though there were other minor betaxanthins from this species which were reported previously (Herbachet al., 2006b). This yellow pigment was identified by the maximum UV adsorption at $480 \mathrm{~nm}$ and principle fragmentation at $m / z 265$, and they eluted earlier than all the betacyanins in the LC chromatogram. 


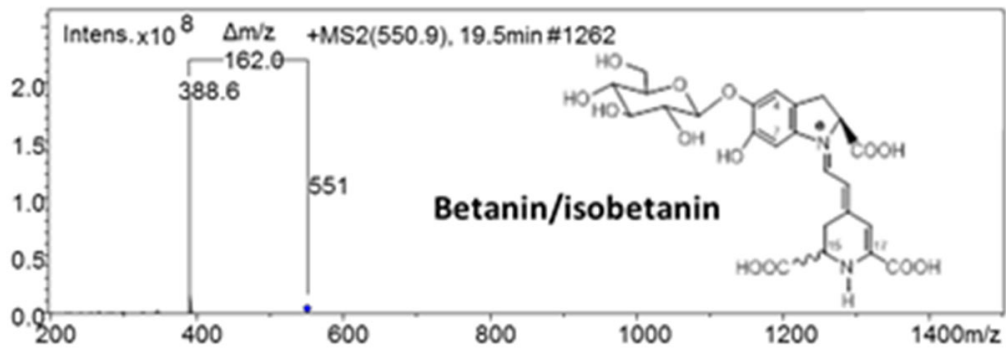

A

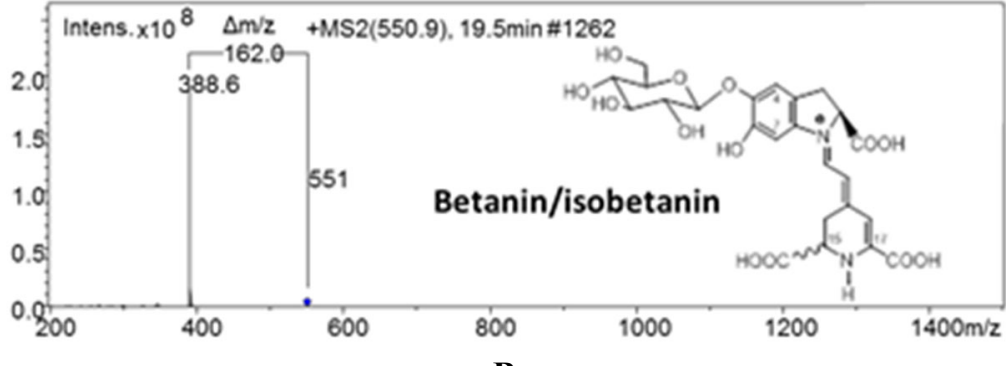

B

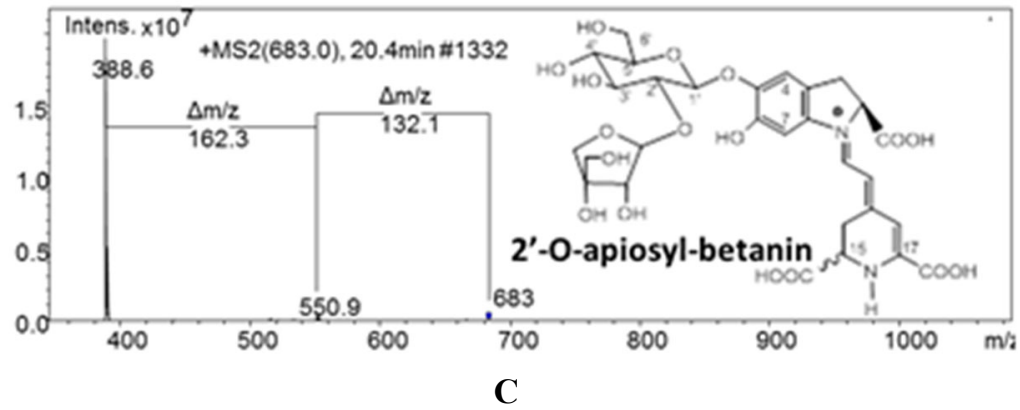

Fig. 4: MS ${ }^{2}$ data, neutral losses and structures of principal betalains from Hylocereus polyhizus

The degraded betacyanins, namely dehydrogenated betanin (or neobetanin) and mono-decarboxy betanin were recognized by the principle daughter ions at $\mathrm{m} / \mathrm{z} 387$ and 349 , respectively. They nominated the neutral losses of a $\Delta m / z 2$ and a $\Delta m / z 44$ (carboxyl group) in comparison with betanin (Figure $6 \mathrm{~A}$ and $6 \mathrm{~B}$ ). The similar degradation products of other pigments were also detected; however, they were found only in traces due to their low concentration (data not shown).

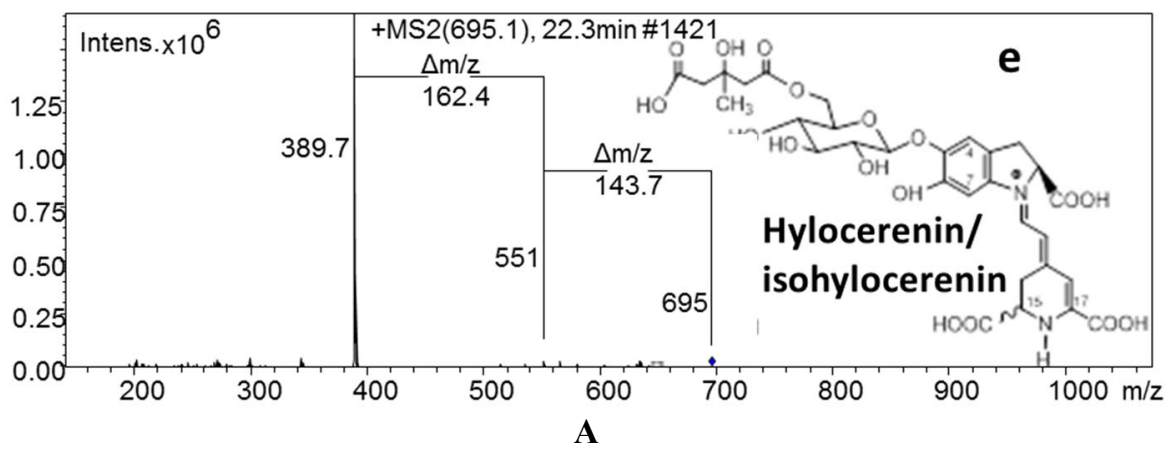




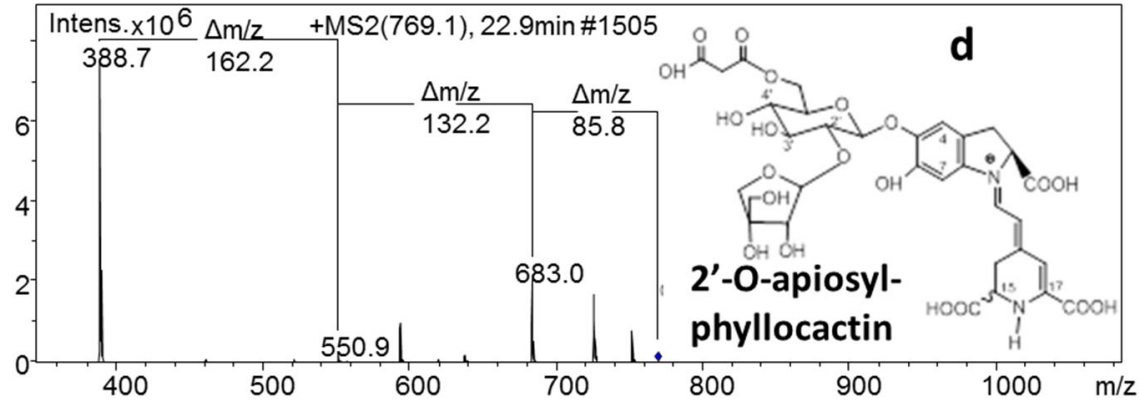

B



Fig. 5: MS-MS data and chemical structures of Vietnamese red dragon fruit betalains
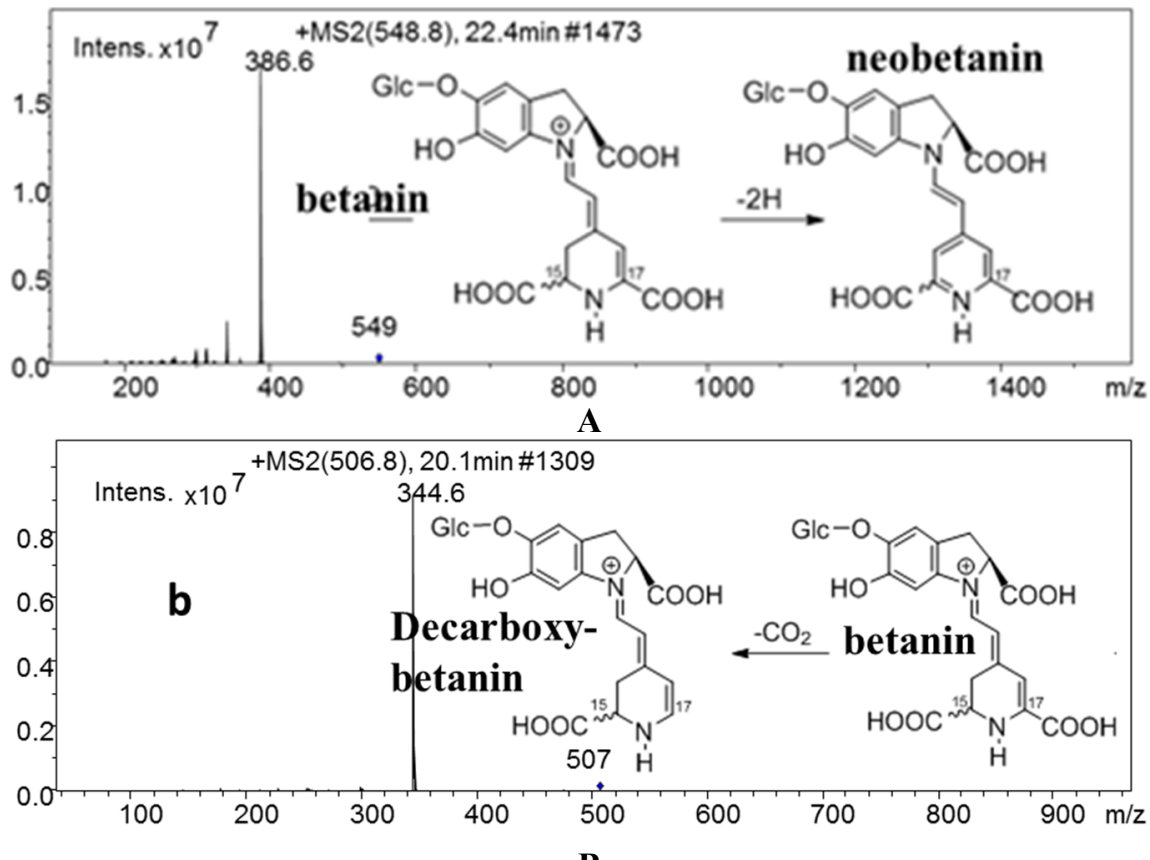

B

Fig. 6: MS-MS data of neobetanin (A) and decarboxy betalains(B) from red dragon fruits

\section{CONCLUSIONS}

This LC-ESI-MS/MS (alternative ionization mode) was applied successfully in characterization of betalains and phenolic compounds (flavonoid glycosides) from several cactus varieties previously. This might be the first report on the mass spectra data of betalains from Vietnamese red dragon fruits. From which betanin epimers $(15 S / 15 R)$ were found as the most dominant compartments next to other minor compounds such as hylocerenin $(15 S / 15 R)$ and phyllocactin $(15 S / 15 R)$. This result not only contributes to the chemical information of this Vietnamese Hylocereus polyhizus cultivar but could also be used for further betalains pigment large scale purification for analytical standards or commercial colorant. 


\section{ACKNOWLEDGMENTS}

The authors would like to thank Prof. Peter Winterhalter - Institute of Food Chemistry - TU Braunschweig for his kind support with allowing the experiments, supplying the devices and chemicals in his laboratory. We wish to thank all the colleagues from the Institute of Food Chemistry - TU Braunschweig for their helps to analyze and interpret the results. Finally, we would send our deepest respect to Dr. Gerold Jerz for his favor on contribution, guidance and supervision this project.

\section{REFERENCES}

Fathordoobady, F., Mirhosseini, H., Salamat, J. and Manap, Y.A., 2016. Effect of solvent type and ratio on betacyanins and antioxidant activity of extracts from Hylocereus polyrhizus flesh and peel by supercritical fluid extraction and solvent extraction. Food Chemistry. 202: 70-80.

Fernández-lópez, J.A., Castellar, R., Obón, J.M. and Almela, L., 2007. Monitoring by liquid chromatography coupled to mass spectrometry the impact of $\mathrm{pH}$ and temperature on the pigment pattern of Cactus Pear fruit extracts. Journal of Chromatography Science. 45(3): 120-125.

He, P.F., Ho, H., Wu, X.X., Hou, M.S. and He, Y.Q., 2012. Bipolaris cactivora causing fruit rot of dragon fruit imported from Vietnam. Plant Pathology \& Quarantine. 2(1): 31-35.

Herbach, K.M., Rohe, M., Stintzing, F.C. and Carle, R., 2006b. Structural and chromatic stability of purple pitaya (Hylocereus polyrhizus [Weber] Britton \& Rose) betacyanins as affected by the juice matrix and selected additives. Food Research International. 39(6): 667-677.

Herbach, K.M., Stintzing, F.C. and Carle, R. 2006a. Stability and color changes of thermally treated betanin, phyllocactin, and hylocerenin solutions. Journal of Agricultural and Food Chemistry. 54(2): 390-398.

Jerz, G., Klose, B., Wybraniec, S., Rodriguez, S.V. and Winterhalter, P., 2013. Preparative separation and pigment profiling of betalains from fruits of Opuntia ficus by ion-pair high-speed countercurrent chromatography (IP-HSCCC) and off-line LC-ESI-MS/MS. In: Bhimanagouda, S.P. (Eds.). Tropical and subtropical fruits: flavors, color, and health benefits. American Chemical Society. Wahsington DC, pp. 3-27.

Le, B.F., Vaillant, F. and Imbert, E., 2006. Pitahaya (Hylocereus spp.): a new fruit crop, a market with a future. Fruits. 61(4): 237-250.

Mabry, T. and Dreiding, A.S., 1968. The betalains: Recent Advances in Phytochemistry. Appleton-Century-Crofts, Division of Meredith Corporation, New York.1: 145-160.
Mac Xuan Hoa, Nguyen Thi Thao Minh, Tran Phan My Duyen, Nguyen Thi Thanh Thao and Dong Thi Thuy, 2017. Microencapsulation of Betacyanin Pigment from Red Flesh Dragon Fruit by Spray Drying. Proceeding of the 35th anniversary of the establishment of Ho Chi Minh City University of Food Industry (1982-2017): 102-108 (in Vietnamese).

Mata, A., Ferreira, J.P., Semedo, C., Serra, T., Duarte, C.M.M. and Bronze, M.R., 2016. Contribution to the characterization of Opuntia spp. juices by LC-DADESI-MS/MS. Food Chemistry. 210: 558-565.

Mereddy, R., Chan, A., Fanning, K., Nirmal, N. and Sultanbawa, Y., 2017. Betalain rich functional extract with reduced salts and nitrate content from red beetroot (Beta vulgaris L.) using membrane separation technology. Food Chemistry. 215: 311-317.

Phan Thi Thanh Que, Nguyen Thi Thu Thuy, Tong Thi Anh Dao and Le Duy Nghia, 2017. Effect of processing and storage condition on the stability of betacyanin in juice of red-fleshed dragon fruit (Hylocereus polyrhizus). Can Tho University Journal of Science. 51(B): 16-23 (in Vietnamese).

Singer, J.W. and Von, E.J.H., 1980. Degradation rates of vulgaxanthine I. Journal of Food Science. 45(3): 489-491.

Stintzing, F.C. and Carle, R., 2007. Betalains - emerging prospects for food scientists. Trends in Food Science and Technology. 18(10): 514-525.

Stintzing, F.C., Herbach, K.M., Mosshammer, M.R., Kugler, F. and Carle, R., 2008. Betalain pigments and color quality. Color Quality of Fresh and Processed Foods. ACS Symposium Series. 983(7): 82-101.

Von, E.J.H., Maing, I.Y. and Amundson, C.H., 1974. Color stability of betanins. Journal of Food Science. 39(2): 334-337.

Wybraniec, S. and Mizrahi, Y., 2002. Fruit flesh betacyanin pigments in Hylocereus Cacti. Journal of Agricultural and Food Chemistry. 50(21): 6086-6089.

Wybraniec, S. and Mizrahi, Y., 2005. Generation of decarboxylated and dehydrogenated betacyanins in thermally treated purified fruit extract from purple pitaya (Hylocereus polyrhizus) monitored by LCMS/MS. Journal of Agricultural and Food Chemistry. 53(17): 6704-6712.

Wybraniec, S., Nowak-Wydra, B., Mitka, K., Kowalski, P. and Mizrahi, Y., 2007. Minor betalains in fruits of Hylocereus species. Phytochemistry. 68(2): 251-259.

Zainoldin, K. and Baba, A., 2009. The effect of Hylocereus polyrhizus and Hylocereus undatus on physicochemical, proteolysis, and antioxidant activity in yogurt. International Journal of Nutrition and Food Engineering. 3(12): 585-590. 